\title{
PRELIMINARY REPORT ON THE USE OF METHONIUM COMPOUNDS AND GENERAL ANAESTHESIA IN OPHTHALMIC SURGERY*
}

\author{
BY \\ B. W. RYCROFT AND G. J. ROMANES \\ From the Plastic Units, East Grinstead and Park Prewett Hospitals
}

IN his lecture "Medicine, Yesterday and To-morrow", Sir Henry Dale mentioned, as the most recent addition to the technique of preparing a patient for a surgical operation,

synthetic substances which, by their action on the sympathetic ganglia, lower the arterial blood pressure and enable a postural adjustment of the patient to produce a practically bloodless field of operation (Dale, 1951).

In routine operations on the eyeball, haemorrhage is of no great consequence, since it may be controlled by careful pre-operative preparation, vasoconstrictor drugs, local pressure, or cautery. But in operations which involve accurate technique in small deep cavities amongst important structures, as in the orbit, severe haemorrhage may be difficult to control, and may impede the smooth progress of the operation.

In Plastic Units this situation frequently occurs, since the ophthalmic surgeon is required to deal with the effects of severe trauma and cicatrization in and around the orbit. Such cases do not lend themselves to local anaesthesia as do most operations on the globe, and general anaesthesia has become routine.

Enderby (1950) reported the use of pentamethonium iodide in the production of controlled hypotension during general anaesthesia, but the results were irregular and unpredictable. Since then he has replaced the drug by hexamethonium bromide and made it possible to achieve accurate temporary control of blood pressure in 85 per cent. of his cases.

Rollason (1951) has also found that hexamethonium is more certain and more effective than pentamethonium iodide.

The object of controlled hypotension or "postural ischaemia" $\dagger$ is to eliminate bleeding at the site of operation. This object is achieved by a combination of the effects of hexamethonium bromide on vessel tone with an arrangement of posture which causes re-distribution of blood to the dependent parts of the body.

Enderby and Pelmore (1951) made the following claims for controlled hypotension by this method in general anaesthesia:

(1) Operations can be done more quickly and more easily.

(2) Blood loss is reduced to a minimum where it is often severe.

* Received for publication August 14, 1951.

+ Sir Henry Dale's phrase. 
(3) Surgical complications such as haematoma and post-operative reactionary oedema are reduced - this is of great advantage in skin graft surgery.

(4) More extensive surgery is possible at one operation when bleeding is no longer a deciding factor.

(5) Post-operative recovery of many patients seems to be improved (see also Rollason, 1951).

Shackleton (1951) later reported further observations on controlled hypotension with methonium compounds and concluded that this was a safe procedure in skilled hands with 75 per cent. good control.

Freeman (1951) suggested that an unexpected failure of hexamethonium hypotension should lead to an investigation of the possible presence of a kidney made ischaemic by local pressure such as might be due to a calcified aneurysm.

\section{Physiology}

Paton (1951) has written that the paralysis of autonomic ganglion transmission may be effected in the following ways:

(1) By interference with the release of acetylcholine at the pre-ganglionic nerve endings, as by procaine injections or local calcium deficiency.

(2) By preventing the acetylcholine from acting on the ganglion cell by the use of some inactive drug i.e., "block by competition". Curare and hexamethonium are examples of such drugs.

(3) By depolarization of the ganglion cell so that it is unaffected by normal acetylcholine, i.e., " block by depolarization". Nicotine produces such an effect but is always preceded by a period of stimulation.

Paton stresses that a drug which is to be used in therapy for blocking a ganglion must be specific: nicotine, on account of the preliminary period of stimulation, is, therefore, unsuitable for use in man. On the other hand, curare blocks the action of acetylcholine on the ganglia but also causes neuro-muscular paralysis and histamine liberation, with consequent confusion of results when a study of blood pressure is under consideration. Wylie and Churchill-Davidson (1951) feel that further investigation of the side-effects of hexamethonium compounds in man are indicated because of their possible action, in large doses, on the motor end-plates: a resemblance to the depolarizing effect of decamethonium has been noted.

Hexamethonium $\left(\mathrm{N}+\left(\mathrm{CH}_{3}\right)_{3} \cdot\left(\mathrm{CH}_{2}\right)_{6} . \mathrm{N}+\left(\mathrm{CH}_{3}\right)_{3}\right)$ is a member of the methonium series of compounds in which the pharmacological activity depends on the number of carbon atoms in the methylene chain joining the terminal quaternary groups. The properties of hexamethonium (C6) are in general those of pentamethonium (C5) and tetramethonium (C4): they have been described in detail by Paton and Zaimis (1951).

In the cat, when the pre-ganglionic nerve is stimulated, contraction of the nictitating membrane, which is served by post-ganglionic fibres, occurs. This preparation has been used by Paton and Zaimis (1951) to assess the effect of ganglionic paralysants. It has been found that hexamethonium will block impulses through the ganglion but that the power of the membrane to contract is still retained as shown by direct injection of adrenaline. 
Similarly, post-ganglionic fibre stimulation also remains effective, and it can also be shown that acetylcholine is released and that the ganglion is not depolarized. Paton, therefore, concludes that hexamethonium affects transmission by producing a ganglionic block. Zaimis has found in rabbits that 60 to 70 per cent. of intravenous dose of hexamethonium di-iodide is excreted unchanged in the urine.

In man, hexamethonium influences blood pressure by release of autonomic tone and not by any direct action on the blood vessels; it has no other depressive effect even in large doses and it has no "atropine-like" or adrenolytic action. Paton emphasizes that the depressor effect of acetylcholine and the pressor effect of adrenaline may be markedly exaggerated when hexamethonium is used (see below). Organe, Paton, and Zaimis (1949) established that in man the effects of the drug were similar to those obtained in the cat, and Arnold and Rosenheim (1949) were able to show that pentamethonium lowered the blood pressure and caused warming of the skin of the lower limbs; adrenaline was also still found to be fully effective in its action on the blood vessels. Burt and Graham (1950) confirmed these findings and reported increased blood flow in the lower limbs. Varying results have been reported in the oral treatment of vascular hypertension by hexamethonium but they are outside the scope of this paper. Milne and Oleesky (1951), studying excretion of hexamethonium compounds in man, found that in people with normal renal function most of an intramuscular dose is excreted in the urine, and that caution in use is necessary where poor renal function has been previously established, since there is then a risk of accumulation of the drug in the blood with prolongation of hypotension.

Enderby (1950) suggested that a patient with a systolic blood pressure of 60-70 mm. Hg produced by hexamethonium and appropriate posture was in a suitable state for certain surgical operations, especially where the operation site was elevated, since bleeding was minimal yet tissue oxygenation and respiration were probably fully preserved. The drug does not cause diminished bleeding alone, but only when used in conjunction with a posture which causes blood to be diverted into the lower limbs. The influence of posture has also to be borne in mind when hexamethonium is used in the oral treatment of hypertension.

Paton concludes with the provocative suggestion that methonium compounds may show further specific influences on ganglion synapses according to their carbon chains, e.g., decamethonium acts only on the myoneural junction, whereas pentamethonium and hexamethonium act only on ganglion synapses.

\section{Clinical Applications}

It has been stated the object of using the drug in conjunction with posture is to obtain a bloodless field for operation. Arteriolar tone is diminished and the veins fill with blood which is then drained by gravity to the dependent 
part of the body: relative ischaemia of the elevated parts of the body is thus produced.

Here the difference in the ischaemia produced by adrenaline must be carefully noted, for it has an important clinical application. With adrenaline the blood clots in the vessel in constriction; the vessel dilates after the effect of the drug has passed off, and this allows the calibre of the vessel to increase, so that the clot may be dislodged by a return of normal blood pressure and a reactionary haemorrhage may occur. With hexamethonium, on the other hand, the vessels clot when dilated; thus subsequent blood pressure changes do not produce reactionary haemorrhage, since there is no increase in calibre of the blood vessel when the effect of the drug wears off: instead there is a return of vessel tone which holds the clot more firmly.

Drops or injections of adrenaline should never be used in conjunction with hexamethonium because of the tendency to obtain exaggerated and dangerous effects. In this connection Burn (1951) points out that adrenaline has a blocking action on the sympathetic ganglia as well as directly on the vessels themselves. Consequently, when the direct vaso-constriction wears off, the ganglion block prevents impulses from the vasomotor centre from reaching the blood vessels, and therefore the blood pressure is not maintained. He suggests that posterior lobe extract of pituitary gland, which has no such block effect on the ganglia, would seem to be the more desirable pressor remedy to use when the blood pressure has fallen low because of the action of hexamethonium. He recommends that it should be administered in such cases as a slow intravenous saline drip containing 10 units pituitary (posterior lobe) extract in each $50 \mathrm{ml}$. saline.

Technique.-Enderby and Pelmore (1951) described their current technique, which is used as a routine in all our ophthalmic cases selected for general anaesthesia in whom this technique is considered suitable. Intra-tracheal anaesthesia is used with thiopentone as the basal anaesthetic. To assist intubation a muscle-relaxant drug is added as required. Anaesthesia is maintained by nitrous oxide and oxygen (2-1) in a semi-closed circle absorber assisted occasionally by very small doses of ether. Cyclopropane is avoided. The Gordh needle is placed in the most convenient vein on the dorsum of the foot.

Dosage.-The initial dose of hexamethonium varies with age and preoperative blood pressure. A young adult receives $50 \mathrm{mg}$. and consecutive blood pressure and pulse readings are taken every 3 minutes. Patients over 40 years receive $30-40 \mathrm{mg}$.; hypertensives and those over 60 years begin with $20 \mathrm{mg}$. Young people show an increased pulse rate of 120-140 per minute and the effect of posture is not marked. In older patients, on the other hand, the pulse rate does not increase much but postural influence is more profound. Therefore, in young people more hexamethonium and extreme posture (up to $40^{\circ}$ reverse Trendelenberg) may be required, whereas in older patients an initial small dose with a small tilt of $5^{\circ}$ to $10^{\circ}$ reverse 
Trendelenberg is often adequate. The object is to maintain a steady systolic level of $60-70 \mathrm{~mm}$. $\mathrm{Hg}$. If the pressure falls below $50 \mathrm{~mm} . \mathrm{Hg}$ the tilt is reduced. Shackleton (1951) reports maintenance of hypotension of $60 \mathrm{~mm}$. Hg for $2 \frac{1}{2} \mathrm{hrs}$ without ill effects during a plastic operation on the scalp.

Precautions.-Enderby and Pelmore stress that all hypotensive patients must have the undivided attention of the anaesthetist, who records consecutive pulse and pressure readings whilst observing the site of operation and regulating the tilt as required. Furthermore, it is very important that there should be a gradual reversion to the horizontal position in the recovery period after operation in order to avoid abrupt variations in the blood pressure. The airway must also be most carefully preserved in hypotension cases. Ivor Lewis (1951) also emphasizes the fundamental importance of a clear airway in this technique. He has reported negligible blood loss in 74 thoracic cases where pressure was maintained at $60 \mathrm{~mm}$. $\mathrm{Hg}$, but in six other cases the blood pressure could not be reduced below $90 \mathrm{~mm} . \mathrm{Hg}$ and there was much greater oozing. Less vomiting was noted and no deleterious effects were encountered in his series.

Similar results are reported by McLoughlin and Watson (1951) in a recent series of 200 plastic operations where low pressure and ischaemia were considered justified on account of the risks of post-operative haematoma formation, serious blood loss, or prolongation of operation time by bleeding, or where a patient had hypertension. They noticed that several patients with a reputation for post-operative vomiting showed an encouraging freedom from this complication. The disadvantages mentioned are the awkward position of the tilted patient and the striking pallor of the skin flaps. Reactionary haemorrhage occurred in two cases, but these workers feel that this will be mitigated in future by improved technique. No serious hazards have been encountered: minor sequelae noted are drowsiness, local venous thrombosis, and anorexia due to reduction in gastric secretion.

Dangers.-Enderby and Pelmore have reported a case where a small reactionary haemorrhage occurred because of an inadequate posture which allowed the blood pressure to rise too quickly after operation. There was post-operative bleeding in two other patients, but in them there were other complicating factors such as disturbance of dressing. In another patient an injection of adrenaline had been given. Vomiting appears to be reduced and occasionally there is temporary disturbance of accommodation after operation. Subject to the precautions stated above, no ill effects have been observed.

Shackleton (1951) lists the following possible dangers in the use of postural hypotension:

(1) Reduction of cerebral circulation below the safe level. In hypertension a sudden reduction could well be dangerous.

(2) An ill effect on the circulation on the kidneys. 
(3) Prolongation of hypotension.

(4) Risk in the immediate post-operative stage, especially during transport and lifting into bed.

(5) A failure to establish initial complete hypotension by an inadequate dose.

He states that these dangers are probably mostly theoretical, but emphasizes the need for keeping accurate blood pressure records on return to the ward and for having "Methedrine" (methyl amphetamine hydrochloride-dosage $10 \mathrm{mg}$.), a potent vaso-constrictor, available as a precaution. He reports a case in which anuria followed the use of the drug and death occurred 2 days after operation. There had been, however, errors of technique in this case, in that the drug was used late to control established haemorrhage instead of at the beginning of the operation to prevent it; there was a possible reduction of blood volume; post-operative hypotension had been allowed to persist too long without correction.

It is essential with this technique to maintain full circulating blood volume and to use an adequate initial dose of the drug to produce an adequate hypotension. Rollason (1951) reports that he has had satisfactory experience and has been much impressed in certain types of operations; he stresses the importance of maintaining blood volume, and avoids vaso-pressors except where the hypotension has been prolonged.

Boyes Korkis (1951) stresses the importance of team-work, and of special attention by the nursing staff to the correct post-operative positioning of the patient in bed. He has found this method of anaesthesia very helpful in major neck surgery, and where the surgeon has to work in a deep dark hole as in external ethmoidectomy.

Use in Ophthalmic Conditions.-It has been our duty to carry out many operations on the orbit in the Plastic Units at East Grinstead and Park Prewett, and we have found this method of anaesthesia to be of great assistance. Such operations include immediate or delayed globe implants of various kinds, especially when associated with large varicose aneurysms of the orbit, cartilage wedge insertions for correction of cyclophoria after middle third facial fractures, reconstruction of the orbit after trauma, rhinostomies of all kinds, neoplasms of the orbit, and different explorations of the orbit. Extensive plastic procedures on the lids, the Blascovicz operation for ptosis, and mucous membrane or skin grafts in socket reconstruction, are much facilitated by the absence of haemorrhage. Our youngest patient was a child aged 3 on whom double cystorhinostomy was performed, and our eldest patient was a hypertensive woman aged 64 who had a large meningioma of the orbit removed after having twice been denied operation on account of the size of the tumour. It is interesting to note that in this patient later a slight blow on the face against a door produced a large haematoma. Both these operations were without incident and bleeding was negligible, and in over one hundred ophthalmic cases we have seen no ill effects. In addition, we have knowledge of over one thousand cases handled by our 
colleagues in the Departments of Anaesthesia at the Plastic Units without any serious complications which could be attributed to this method of anaesthesia.

During extensive operations on the orbit which would normally result in considerable haemorrhage, it is uncanny to see no bleeding from the incision; no sucker is necessary and no artery forceps are used when postural hypotension has been established. The detailed dissection of structures is easy and the definition of fine detail in the orbit can be carried out with ease. In rhinostomies, troublesome bone bleeding is eliminated, and accurate suture work at depth is much facilitated. It is unusual for us to have to use more than one or two swabs in extensive operations such as the removal of large orbital tumours. Furthermore, it is our opinion that post-operative fibrosis from haemorrhage, and oedema as the result of manipulation, are much reduced.

Intra-Ocular Pressure.-We feel that this method of anaesthesia might be useful in the treatment of acute glaucoma. As is well known, many such cases respond to treatment by the usual local remedies combined with repeated retrobulbar injections of procaine and alcohol. There are, however, a few stubborn cases in which the intra-ocular pressure remains dangerously and persistently high, so that operation under general anaesthesia is desirable. Some small reduction of the eye pressure can be expected with basal anaesthesia, but when hexamethonium is used the fall in intra-ocular pressure may be considerable, and surgical procedures such as basal iridectomy can be carried out with greater safety.

In the normal eye, it can be demonstrated that the intra-ocular pressure may fall from 25 to $18 \mathrm{~mm}$. $\mathrm{Hg}$ under basal anaesthesia, but with postural hypotension the intra-ocular pressure cannot be registered on the Schiötz tonometer and the cornea will remain concave until it is manipulated back into shape.

Much more experience in the application of the drug in this condition is required, but the suggestion is offered in this preliminary report in order that it may be tried by colleagues who may require general anaesthesia in stubborn cases of acute glaucoma.

\section{Discussion}

It is our cautious and preliminary opinion that there is a place for the use of hexamethonium compounds in general anaesthesia for ophthalmic surgery.

Ophthalmic operations are accurate procedures which demand exact technique, and any agent which is safe should be used if it adds to the ease and precision of the surgeon's task. The value of an ischaemic field in orthopaedic surgery has long been recognized. With combined hypotension and postural ischaemia, similar benefits are now extended to the ophthalmic field. The operations mentioned above are not impossible without this 
agent, but all are rendered easier and more accurate with it. Much more experience is yet required, but in our ophthalmic surgery so far the value of the drug has been unquestionable, and it has reduced many surgical manœuvres of potential difficulty to relative simplicity.

\section{Summary}

(1) A bloodless field of operation can be produced by the use of hexamethonium with a suitable posture for operations in and around the orbit where bleeding would impair efficient and accurate surgery. It is a safe procedure in skilled hands.

(2) The undivided attention of a skilled anaesthetist is essential both during the operation and until normal blood pressure has been established on return to bed. Adrenaline in all forms is contraindicated. The blood volume must be maintained.

(3) No serious ill effects have been encountered in our series of ophthalmic cases, nor reported in a series of one thousand cases at the two Plastic Units which we serve.

(4) This method has a definite potential value in the treatment of severe acute glaucoma requiring surgical treatment under general anaesthesia.

(5) Other advantages are that the assistant is freed from the task of maintaining haemostasis, that fewer instruments are employed in a small wound, and that considerable time can be saved.

(6) Blood loss is eliminated, post-operative oedema due to manipulation is reduced, and secondary fibrosis from organized blood clots, especially important in rhinostomies, is avoided.

We acknowledge with gratitude the help and guidance we have received from Dr. Hale Enderby who has been largely responsible for the introduction of this method of anaesthesia. Our thanks are also due to other colleagues in the Departments of Anaesthesia at the Plastic Units at East Grinstead and Park Prewett Hospitals with whom we have the privilege to work.

\section{REFERENCES}

Arnold, P., and Rosenheim, M. L. (1949). Lancet, 2, 321.

BURN, J. H. (1951). Ibid., 1, 642.

Burt, C. C., and Graham, A. J. P. (1950). Brit. med. J., 1, 455.

DALE, SIR HENRY (1951). Ibid., $2,4$.

ENDERBY, G. E. H. (1950). Lancet, 1, 1145.

and Pelmore, J. F. (1951), Ibid., 1, 663.

FreEMAN, Z. (1951). Brit. med. J., 1, 1079.

KORKIS, F. BOYES (1951). Lancet, 1, 801.

LEWIS, I. (1951). Ibid., 2, 150.

McLaughuin, C. R., and Watson, J. (1951). Brit. med. J., 4; 121.

MILNE, G. E., and OLEESKY, S. (1951). Lancet, 1, 889.

ORgane, G., PATON, W. D. M., and Zaimis, E. J. (1949). Ibid., 1, 21.

PATON, W. D. M. (1951). Brit. med. J., 1, 773.

and ZAIMIS, E. J. (1951). Brit. J. Pharmacol., 6, 155.

Rollason, W. N. (1951). Brit. med.J., 1, 1447.

ShaCKLeton, R. P. W. (1951). Ibid., 1, 1054.

WYLIE, W. D., and ChuRChILl-DAVIDSON, H. C. (1951). Ibid., $2,54$. 